$\square$ Math-Net.Ru

Общероссийский математический портал

П. Б. Уткин, Гипотеза о размерности для квадрик кораз- 
мерности 3 и более, Матем. заметки, 2002, том 72, выпуск $1,152-156$

DOI: https://doi.org/10.4213/mzm660

Использование Общероссийского математического портала Math-Net.Ru подразумевает, что вы прочитали и согласны с пользовательским соглашением http://www. mathnet.ru/rus/agreement

Параметры загрузки:

IP: 34.239 .49 .27 
26 апреля 2023 г., 18:31:35

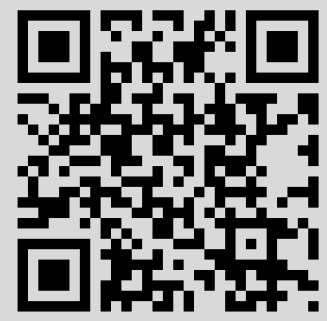




\section{ГИПОТЕЗА О РАЗМЕРНОСТИ ДЛЯ КВАДРИК КОРАЗМЕРНОСТИ 3 И БОЛЕЕ}

\section{П. Б. Уткин}

1. Введение. Пусть $\boldsymbol{z}=\left(z^{i}\right), i \in 1, \ldots, n ; \boldsymbol{w}=\left(w^{j}\right), j \in 1, \ldots, k,-$ координаты в $\mathbb{C}^{n+k}$; $\langle\boldsymbol{z}, \overline{\boldsymbol{z}}\rangle=\left(\langle\boldsymbol{z}, \overline{\boldsymbol{z}}\rangle^{1}, \ldots,\langle\boldsymbol{z}, \overline{\boldsymbol{z}}\rangle^{k}\right)-$ векторозначная эрмитова форма, задаваемая при помощи $k$ эрмитовых матриц. Вещественно аналитическое многообразие $M$ в $\mathbb{C}^{n+k}$, задаваемое уравнением $\operatorname{Im} \boldsymbol{w}=\langle\boldsymbol{z}, \overline{\boldsymbol{z}}\rangle$, называется $(n, k)$-квадрикой. Эти алгебраические поверхности играют в теории вещественных подмногообразий комплексного пространства особую роль. Как показано в [1], квадрика $M$ является поверхностью типа $(n, k)$ с самой богатой группой голоморфных автоморфизмов. Этим обусловлен интерес к описанию этой группы и соответствующей ей алгебры Ли.

Группа автоморфизмов квадрики конечномерна тогда и только тогда, когда квадрика невырождена в следующем смысле [2].

Работа выполнена при финансовой поддержке Российского фонда фундаментальных исследований, грант № 96-01-96185. 
ОПРЕДЕЛЕНИЕ 1. $(n, k)$ квадрика назьвается невырожденной если вьполнены два условия:

1) $\langle\boldsymbol{z}, \overline{\boldsymbol{z}}\rangle^{1}, \ldots,\langle\boldsymbol{z}, \overline{\boldsymbol{z}}\rangle^{k}$ линейно независимы.

2) $\forall \boldsymbol{e} \in \mathbb{C}^{n}:\left(\forall \boldsymbol{z} \in \mathbb{C}^{n}:\langle\boldsymbol{z}, \overline{\boldsymbol{e}}\rangle=0\right) \rightarrow(\boldsymbol{e}=0)$.

Пусть невырожденная квадрика $M$ задается набором $k$ эрмитовых матриц $\boldsymbol{H}_{i}, i \in 1 \ldots k$; тогда условие 1) означает линейную независимость матриц $\boldsymbol{H}_{i}$, а условие 2$) \bigcap_{i \in 1, \ldots, k} \operatorname{Ker}\left(\boldsymbol{H}_{i}\right)=\varnothing$.

Квадрика однородна, на ней транзитивно действует подгруппа автоморфизмов, состоящая из аффиных преобразований вида

$$
z \mapsto z+p, \quad w \mapsto w+q+2 i\langle z, \bar{p}\rangle, \quad(p, q) \in M .
$$

Подгруппа группы автоморфизмов, сохраняющих начало координат, не имеет столь простого и явного описания, однако имеется следующее описание соответствующей ей алгебры $g(M)[1]$ :

$$
\forall h \in g(M): h=f \frac{\partial}{\partial \boldsymbol{z}}+g \frac{\partial}{\partial \boldsymbol{w}}
$$

где

$$
\begin{gathered}
f=C \boldsymbol{z}+A(\boldsymbol{z}, \boldsymbol{z})+a \boldsymbol{w}+B(\boldsymbol{z}, \boldsymbol{w}) \\
g=s \boldsymbol{w}+2 i\langle\boldsymbol{z}, \bar{a} \boldsymbol{w}\rangle+r(\boldsymbol{w}, \boldsymbol{w})
\end{gathered}
$$

Соответственно $a: \mathbb{C}^{k} \rightarrow \mathbb{C}^{n}, C: \mathbb{C}^{n} \rightarrow \mathbb{C}^{n}, s: \mathbb{C}^{k} \rightarrow \mathbb{C}^{k}$ - линейные отображения, а $A: \mathbb{C}^{n} \times C^{n}$ $\rightarrow \mathbb{C}^{n}, B: \mathbb{C}^{n} \times C^{k} \rightarrow \mathbb{C}^{n}, r: \mathbb{C}^{k} \times C^{k} \rightarrow \mathbb{C}^{k}-$ билинейные отображения, удовлетворяющие системе уравнений

$$
\begin{gathered}
2 \operatorname{Re}(\langle C \boldsymbol{z}, \overline{\boldsymbol{z}}\rangle)=s\langle\boldsymbol{z}, \overline{\boldsymbol{z}}\rangle, \\
\langle A(\boldsymbol{z}, \boldsymbol{z}), \boldsymbol{z}\rangle=2 i\langle\boldsymbol{z}, \bar{a}\langle\boldsymbol{z}, \overline{\boldsymbol{z}}\rangle\rangle, \\
\operatorname{Re}\langle B(\boldsymbol{z}, \boldsymbol{u}), \boldsymbol{z}\rangle=r(\langle\boldsymbol{z}, \overline{\boldsymbol{z}}\rangle, \boldsymbol{u}), \\
\operatorname{Im}\langle B(\boldsymbol{z},\langle\boldsymbol{z}, \overline{\boldsymbol{z}}\rangle), \boldsymbol{z}\rangle=0,
\end{gathered}
$$

причем $r, s$ вещественны.

Если ввести естественную градуировку на $g(M)$, а именно вес $\boldsymbol{z}$ равен 1 , вес $\boldsymbol{w}$ равен 2 , то $g(M)$ распадется на три однородные компоненты $g_{0}(M), g_{1}(M), g_{2}(M)$ (веса $\partial / \partial \boldsymbol{z}$ и $\partial / \partial \boldsymbol{w}$ определяются как -1 и -2 соответственно).

При $3 \leqslant k \leqslant n^{2}-3$ квадрики обладают эфффектом “жесткости” [1], [3]: для квадрики общего положения компоненты $g_{1}$ и $g_{2}$ отсутствуют (равны нулю), а $g_{0}$ двумерна, что соответствует мультипликативной группе комплексных чисел, которая действует скалярными растяжениями $z \mapsto \lambda z$, $w \mapsto|\lambda|^{2} w$. Однако это не мешает существованию исключительных квадрик с весьма богатьми группами (см. например [4], [5]). Во всех имевшихся примерах выполнялись оценки $\operatorname{dim}\left(g_{1}(M)\right)$ $\leqslant 2 n, \operatorname{dim}\left(g_{2}(M)\right) \leqslant k$, сформулированные в [6] в качестве гипотезы. В данной работе приведен контрпример к данной гипотезе, а также описаны квадрики коразмерности $3 \mathrm{c} \operatorname{dim}\left(g_{1}(M)\right)>2 n$. В качестве следствия описания получено подтверждение гипотезы $\operatorname{dim}\left(g_{1}(M)\right) \leqslant 2 n$ для квадрик типа $(4,3)$ (то, что это так для типа $(3,3)$ было известно и раньше [4]).

\section{2. Контрпример}

2.1. Коразмерность 3. Рассмотрим $(3, n)$-квадрику $M$, заданную тремя эрмитовыми матрицами

$$
\begin{gathered}
H_{1}=\operatorname{diag}\left(\varepsilon_{1}, \ldots, \varepsilon_{m}, \varepsilon_{m+1} I, \ldots, \varepsilon_{m+t} I\right), \quad H_{2}=\operatorname{diag}(\underbrace{0, \ldots, 0}_{m}, \varepsilon_{m+1} J, \ldots, \varepsilon_{m+t} J), \\
H_{3}=\operatorname{diag}(\underbrace{0, \ldots, 0}_{m}, H_{3}^{\prime}) ;
\end{gathered}
$$


матрица $H_{3}^{\prime}$ эрмитова и все ее ненулевые элементы стоят на местах пересечения столбцов с нечетными номерами и строк с нечетными номерами. Назовем такие квадрики $\alpha$-квадриками. Далее,

$$
\begin{gathered}
H_{1}=\operatorname{diag}\left(\varepsilon_{1}, \ldots, \varepsilon_{m}, \varepsilon_{m+1} I, \ldots, \varepsilon_{m+t} I\right), \quad H_{2}=\operatorname{diag}(\underbrace{0, \ldots, 0}_{m}, \varepsilon_{m+1} J, \ldots, \varepsilon_{m+t} J), \\
H_{3}=\left(\begin{array}{cc}
H_{3}^{1} & C \\
C^{*} & H_{3}^{\prime}
\end{array}\right), \quad H_{1}^{1}=\operatorname{diag}\left(\varepsilon_{1}, \ldots, \varepsilon_{m}\right) \rightarrow H_{3}^{1} H_{1}^{1} H_{3}^{1}=0, \quad H_{3} H_{1} H_{3}=0
\end{gathered}
$$

матрица $H_{3}^{\prime}$ как у $\alpha$-квадрики, причем

$$
I=\left(\begin{array}{ll}
0 & 1 \\
1 & 0
\end{array}\right), \quad J=\left(\begin{array}{ll}
1 & 0 \\
0 & 0
\end{array}\right), \quad \varepsilon_{i}= \pm 1
$$

Такие квадрики будем называть $\beta$-квадриками. Всякая $\alpha$-квадрика является $\beta$-квадрикой, их выделение продиктовано тем, что они имеют наибольшую размерность подалгебры $g_{1}$.

Tеорема. 1) Eсли $\operatorname{dim}\left(g_{1}\right) \geqslant 2 n$, то $M$ әквивалентна $\beta$-квадрике.

2) Для $\alpha$-квадрик $\operatorname{dim}\left(g_{1}\right)=4(m+t)>2 n=2 m+4 t, \operatorname{dim}\left(g_{2}\right)=4>k=3$.

3) Д几я $\beta$-квадрик $\operatorname{dim}\left(g_{1}\right) \leqslant 4(m+t)$.

4) Невырожденные $\alpha$-квадрики существуют только при $n \geqslant 5$; для невырожденных $\beta$-квадрик при $n=4 \operatorname{dim}\left(g_{1}\right) \leqslant 2 n=8$, в частности, для любой $(3,4)$ квадрики $\operatorname{dim}\left(g_{1}\right) \leqslant 2 n=8$.

Теорема доказьвается решением уравнения (0.2) с использованием приведения двух эрмитовых матриц к каноническому виду [7] и отбрасьванием случаев с низкой размерностью $g_{1}$.

Для $\alpha$-квадрик можно явно выписать $g_{1}, g_{2}: g_{1}$ задается линейным отображением $a: \mathbb{C}^{k} \rightarrow \mathbb{C}^{n}$, a $g_{2}$ билинейным отображением $r_{1}: \mathbb{C}^{k} \times \mathbb{C}^{k} \rightarrow \mathbb{C}$ и произвольным вещественньм числом $b$.

А именно,

$$
\begin{gathered}
g_{1}=(A(\boldsymbol{z}, \boldsymbol{z})+a \boldsymbol{w}) \frac{\partial}{\partial \boldsymbol{z}}+2 i\langle\boldsymbol{z}, \bar{a} \boldsymbol{w}\rangle \frac{\partial}{\partial \boldsymbol{w}}, \\
a=\left\|a_{1}, a_{2}, a_{3}\right\|, \quad a_{1}=0, \quad \boldsymbol{H}_{2} a_{2}=\boldsymbol{H}_{2} a_{3}=0 .
\end{gathered}
$$

$A$ определяется из системы

$$
A^{t}(\boldsymbol{z}, \boldsymbol{z}) h_{i}^{j}=\boldsymbol{z}^{t} \boldsymbol{H}_{i}(2 i \bar{a}) \boldsymbol{G}_{j} \boldsymbol{z}
$$

где $h_{i}^{j}-j$ столбец матрицы $\boldsymbol{H}_{i}$, a $\boldsymbol{G}_{j}^{t}=\left\|h_{1}^{j}, h_{2}^{j}, h_{3}^{j}\right\|, i=1,2,3, j \in 1, \ldots, n$. Данная система есть уравнение (0.2) и является совместной для таких $a$. Обозначив $v^{j}(\boldsymbol{z}, \boldsymbol{z})=\boldsymbol{z}^{t} \boldsymbol{H}_{1}(2 i \bar{a}) \boldsymbol{G}_{j} \boldsymbol{z}$, получим $A(\boldsymbol{z}, \boldsymbol{z})=\boldsymbol{H}_{1} v$.

Для $g_{2}(M)$ через $r^{1}(\boldsymbol{w}, \boldsymbol{w})$ обозначается первая координата $r(\boldsymbol{w}, \boldsymbol{w})$. Она должна иметь вид

$$
r_{1}=\left\|\begin{array}{lll}
0 & 0 & 0 \\
0 & d & e \\
0 & e & c
\end{array}\right\|, \quad d, e, c \in \mathbb{R}
$$

Остальные две координаты $r(\boldsymbol{w}, \boldsymbol{w})$ нулевые; если $B(\boldsymbol{z}, \boldsymbol{w})=B(\boldsymbol{w}) \boldsymbol{z}$, то

$$
B(\boldsymbol{w})=\left(d w^{2}+e w^{3}+i b w^{3}\right) \boldsymbol{H}_{1} \boldsymbol{H}_{2}+\left(e w^{2}+c w^{3}-i b w^{2}\right) \boldsymbol{H}_{1} \overline{\boldsymbol{H}}_{3}
$$


2.2. Коразмерность больше 3. $\alpha$-квадрики очевидньм образом распространяются на большие коразмерности, а именно матрицы $\boldsymbol{H}_{1}, \boldsymbol{H}_{2}$ остаются прежними а матрицы $\boldsymbol{H}_{3}, \ldots, \boldsymbol{H}_{k}$ делаются типа $\boldsymbol{H}_{3}$. Тогда в $g_{1}$ содержится линейное подпространство полей вида

$$
\begin{gathered}
g_{1}=(A(\boldsymbol{z}, \boldsymbol{z})+a \boldsymbol{w}) \frac{\partial}{\partial \boldsymbol{z}}+2 i\langle\boldsymbol{z}, \bar{a} \boldsymbol{w}\rangle \frac{\partial}{\partial \boldsymbol{w}}, \\
a=\left\|a_{1} \ldots a_{k}\right\|, \quad a_{1}=0, \quad \boldsymbol{H}_{2} a_{i}=0, \quad i \in 1, \ldots, k, \\
\boldsymbol{G}_{j}^{t}=\left\|h_{1}^{j} \ldots h_{k}^{j}\right\| .
\end{gathered}
$$

Далее $A$ определяется из системы (1.1), где $i \in 1, \ldots, k$, а $j$ определяется, как и в случае коразмерности $k=3$. Выделение данного подпространства дает нам оценку снизу:

$$
\operatorname{dim}\left(g_{1}\right) \geqslant 2(k-1)(m+t) .
$$

Таким же образом в $g_{2}$ выделяется подпространство полей, определяемых из $r^{1}(\boldsymbol{w}, \boldsymbol{w})$ и из произвольной косоэрмитовой матрицы $b=\left\|b_{j}^{i}\right\|, i, j \in 2, \ldots, k$. Если

$$
B(\boldsymbol{z}, \boldsymbol{w})=B(\boldsymbol{w}) \boldsymbol{z}
$$

то

$$
B^{t}(\boldsymbol{w})=\sum_{j=2, \ldots, k} r_{j}^{1}(\boldsymbol{w}) \boldsymbol{H}_{j} \boldsymbol{H}_{1}+\sum_{i, j=2, \ldots, k} b_{i}^{j} w^{i} \boldsymbol{H}_{j} \boldsymbol{H}_{1}
$$

где

$$
r^{1}(\boldsymbol{w}, \boldsymbol{w})=\left\|\begin{array}{c}
r_{1}^{1}(\boldsymbol{w}, \boldsymbol{w}) \\
\vdots \\
r_{k}^{1}(\boldsymbol{w}, \boldsymbol{w})
\end{array}\right\| .
$$

Это дает нам оценку $\operatorname{dim}\left(g_{2}\right) \geqslant(k-1)^{2}$. Точных равенств нет, поскольку выше выписаны лишш подпространства, а не сами $g_{1}, g_{2}$.

\section{3. Подгруппа нелинейных автоморфизмов}

Подгруппа нелинейньг голоморфных автоморфизмов $M$, за которую отвечает подалгебра $g_{1}+g_{2}$ для случая коразмерности 3 и квадрик из примера 1 , может быть явно получена путем вычисления соответствующих однопараметрических подгрупп. Система дифференциальных уравнений имеет специфический вид и позволяет последовательно вычислить все координатные функции.

Точнее, система будет такой:

$$
\begin{gathered}
\dot{z}^{1}=a_{2}^{1} w^{2}+a_{3}^{1} w^{3}, \\
\vdots \\
\dot{z}^{m}=a_{2}^{m} w^{2}+a_{3}^{m} w^{3}, \\
\dot{z}^{m+2 i-1}=0, \\
\dot{z}^{m+2 i}=F_{i}\left(w^{2}, w^{3}, z^{1}, \ldots, z^{m}, z^{m+2 j-1}\right), \\
\dot{w}^{1}=F_{t+1}\left(w^{2}, w^{3}, z^{1}, \ldots, z^{n}\right), \\
\dot{w}^{2}=0, \\
\dot{w}^{3}=0,
\end{gathered}
$$

где $i \in 1, \ldots, t, j \in 1, \ldots, t$. Соответственно $F_{1}, \ldots, F_{t+1}$ - квадратные многочлены, определяемые из $a(\boldsymbol{w}), r(\boldsymbol{w}, \boldsymbol{w}), B(\boldsymbol{z}, \boldsymbol{w})$, участвующие в уравнениях $(0.2),(0.3),(0.4) ;$ коэфффициенты $a_{i}^{j}-$ из $a(\boldsymbol{w})$. В этом случае решение получается полиномиальным степени 2 . 
Решим эту систему. Для удобства запишем $z=\left(z^{1}, \ldots, z^{m}, z^{m+1}, \ldots, z^{m+2 t}\right)$ в виде $z=$ $\left(z_{1}^{1}, \ldots, z_{1}^{m}, z_{2}^{1}, z_{3}^{1}, \ldots, z_{2}^{t}, z_{3}^{t}\right)$. Соответственно $A=\left(A_{1}, A_{2}, A_{3}\right), a=\left(a_{1}, a_{2}, a_{3}\right), B=\left(B_{1}, B_{2}\right.$, $\left.B_{3}\right) ;$ тогда $z=z_{1}+z_{2}+z_{3}$ и экспонента данного поля даст автоморфизм

$$
\begin{aligned}
z_{1} & \rightarrow z_{1}+a_{1} w \\
z_{2} & \rightarrow z_{2} \\
z_{3} & \rightarrow z_{3}+a_{3} w+A_{3}\left(z_{2}, z_{2}\right)+A_{3}\left(z_{1}, z_{2}\right)+B_{3}(z, w) \\
w^{1} & \rightarrow w^{1}+r^{1}(w, w)+2 i\left\langle z_{1}, \bar{a} w\right\rangle^{1}+i\left\langle z_{1}, \bar{a} w\right\rangle^{1} \\
w^{2} & \rightarrow w^{2} \\
w^{3} & \rightarrow w^{3}
\end{aligned}
$$

Все участвующие отображения $a, A, r, B$ из записи подалгебры $g_{1}+g_{2}$ :

$$
(a w+A(z, z)+B(z, w)) \frac{\partial}{\partial z}+(r(w, w)+2 i\langle z, \bar{a} w\rangle) \frac{\partial}{\partial w} .
$$

\section{СПИСОК ЦИТИРОВАННОЙ ЛИТЕРАТУРЫ}

1. Белошапка В. К. // Матем. сб. 1991. № 2. С. 203-219. 2. Белошапка В. К. // Изв. АН CCCP. Cер. матем. 1988. T. 52 № 2. C. 437-442. 3. Ezhov V., Schmalz G. // Math. Nachrichten. 1999. V. 204. Р. 41-60. 4. Палинчак Н. Ф. // Матем. заметки. 1994. Т. 55. № 5. С. 110-115. 5. Анисова Е. Г. // Матем. заметки. 1998. Т. 64. №6. С. 803-811. 6. Beloshapka V. K. // Russian J. Math. Phys. 1996. V. 4. №3. Р. 393-396. 7. Шевченко С. Н. // Изв. РАН. Сер. матем. 1994. T. 58. № 4. C. 149-172. 